\title{
Desogestrel plus testosterone effectively suppresses spermatogenesis but also causes modest weight gain and high- density lipoprotein suppression
}

Received August 26, 1999; revised and accepted April 18, 2000.

Supported by Grant CONRAD CSA-95-165 from the Andrew W. Mellon Foundation; National Institutes of Health Grant HD 12629; and Department of Veterans Affairs Research Funds.

Reprint requests: Bradley D. Anawalt, M.D., S-111 VAPSHCS, 1660 South Columbian Way, Seattle, Washington 98108 (FAX: 206-764-2689).

a Department of Medicine, University of Washington.

${ }^{b}$ Geriatric Research, Education and Clinical Center, Veterans Affairs Puget Sound Health Care System, University of Washington.

${ }^{c}$ NV Organon.

d Population Center for Research in Reproduction, Department of Medicine, University of Washington.

0015-0282/00/\$20.00 PII S0015-0282(00)01490-4

\author{
Bradley D. Anawalt, M.D., ${ }^{a}$ Karen L. Herbst, M.D., Ph.D., ${ }^{a}$ \\ Alvin M. Matsumoto, M.D., ${ }^{\mathrm{a}, \mathrm{b}}$ Titia M. T. Mulders, Ph.D., ${ }^{\mathrm{c}}$ \\ Herjan J. T. Coelingh-Bennink, M.D., Ph.D., ${ }^{c}$ and William J. Bremner, M.D., Ph.D. ${ }^{\text {a,d }}$ \\ University of Washington, Seattle, Washington; and NV Organon, Oss, The Netherlands
}

Objective: To test the hypothesis that oral desogestrel (DSG) plus testosterone would uniformly and rapidly suppress sperm concentrations in young men as effectively as levonorgestrel (LNG) plus testosterone and cause less high-density lipoprotein (HDL) suppression and weight gain.

Design: Single-blind, randomized trial.

Setting: VA Puget Sound and University of Washington, Seattle, Washington.

Patient(s): Twenty-four healthy young men, aged 20-49.

Intervention(s): Subjects were randomized to three groups of men who were administered 6 months of therapy with oral DSG plus im testosterone enanthate: $150 \mu \mathrm{g}$ of DSG plus $50 \mathrm{mg}$ of testosterone (DSG 150-T 50), $150 \mu \mathrm{g}$ of DSG plus $100 \mathrm{mg}$ of testosterone (DSG 150-T 100) or $300 \mu \mathrm{g}$ of DSG plus $100 \mathrm{mg}$ of testosterone (DSG 300-T 100). We compared these three groups to two groups of historical controls of 100 $\mathrm{mg}$ of im testosterone alone or $150 \mu \mathrm{g}$ of oral LNG plus $100 \mathrm{mg}$ of im testosterone (LNG 125-T 100 group) enrolled in similar studies.

Main Outcome Measure(s): Suppression of sperm counts to severe oligoazoospermia (sperm counts $<1 \times$ $10 \% \mathrm{~mL}$ ) and azoospermia, weight gain, and serum high-density cholesterol (HDL) suppression.

Result(s): Azoospermia was achieved in all eight men receiving DSG 150-T 100 and seven of the eight men in the DSG 300-T 100 group. DSG 150 or 300 plus T 100 suppressed spermatogenesis as effectively as LNG 125-T 100 and more effectively than DSG 50-T 100 or testosterone alone. All groups tended to gain weight compared with their baseline, but the weight gain was greatest (and statistically significant) in the DSG 150-T 100, DSG 300-T 100, and LNG 125-T 100 groups. Serum HDL levels were modestly suppressed in all groups, and this effect was greatest in the DSG 300-T 100 and LNG 125-T 100 groups.

Conclusion(s): The combination of DSG plus testosterone is a very effective regimen for suppression of spermatogenesis and has acceptably low side effects. (Fertil Steril ${ }^{\circledR}$ 2000;74:707-14. (C2000 by American Society for Reproductive Medicine.)

Key Words: Male hormonal contraception, spermatogenesis, oligoazoospermia, azoospermia, testosterone, gonadotropins, desogestrel

The administration of high dosages of exogenous testosterone or testosterone plus a progestin can suppress circulating gonadotropins to near undetectable levels and sperm concentrations to azoospermia or to severe oligoazoospermia $\left(<1.0 \times 10^{6} \mathrm{sperm} / \mathrm{mL}\right)$ in normal, healthy men (1-3). In the World Health Organization (WHO) efficacy trial of the contraceptive effectiveness of high-dosage IM testosterone $(200 \mathrm{mg} / \mathrm{wk})$ in normal men, the pregnancy rates per 100 person-years were $0 \%$ in the men whose sperm counts suppressed to azoospermia and $1 \%$ in the men whose sperm counts suppressed to $<1 \times 10^{6} / \mathrm{mL}$. These failure rates compare favorably to rates of reversible female methods, such as injectable and oral hormonal contraceptives $(0.3$ and 3 per 100 person-years) and the most common reversible male method, the condom (12 per 100 person-years) $(3,4)$. However, administration of high-dosage exogenous testosterone (200 $\mathrm{mg} / \mathrm{wk}$ ) alone does not uniformly suppress 
spermatogenesis, it causes weight gain, and tends to suppress high-density lipoprotein (HDL) cholesterol levels (5).

More recently, combination regimens of lower dosages of progestins plus androgens have been investigated and seem to be promising (6). We previously showed that the administration of high-dosage oral levonorgestrel (LNG, 250 or $500 \mu \mathrm{g} / \mathrm{d}$ ) plus testosterone enanthate (100 mg/wk IM) to normal young men was more rapidly and uniformly effective in achieving severe oligoazoospermia or azoospermia than exogenous testosterone alone. However, the combination of high-dosage LNG plus testosterone caused significant suppression of serum HDL cholesterol levels and weight gain (7). A lower-dosage LNG (125 $\mu \mathrm{g} / \mathrm{d}$ orally) plus testosterone (100 mg/wk) regimen was as effective as the higher-dosage LNG plus testosterone regimens in achieving severe oligoazoospermia or azoospermia, but lower-dosage LNG plus testosterone caused less suppression of HDL cholesterol levels and less weight gain (8).

Although the combination of lower-dosage LNG plus testosterone seems to be a promising possible male contraceptive regimen, there has been interest in using combinations of less androgenic progestins plus T. Desogestrel (DSG), a potent synthetic progestin that has been used in recently developed female oral contraceptive pills, may cause less HDL suppression (9). In this study, we tested the hypothesis that oral DSG plus low-dosage testosterone would uniformly and rapidly suppress sperm concentrations in young men as effectively as oral low-dosage LNG plus low-dosage testosterone without causing HDL suppression or weight gain.

\section{MATERIALS AND METHODS}

\section{Subjects}

Normal men, aged 20-49 years, were recruited by advertisement on bulletin boards, in newspapers, and on the radio. Inclusion criteria were a normal medical history and physical examination; the absence of current use of prescription medications; normal basal serum testosterone, FSH, and LH levels; three successive normal seminal fluid analyses (sperm count $>20 \times 10^{6} / \mathrm{mL}$ and motility and oval forms $>50 \%$ after 48 hours of ejaculatory abstinence) on specimens collected at 2-week intervals; and normal values on routine hematology, blood chemistry, urinalysis, and fasting lipid profiles. Exclusion criteria included any history of significant acute or chronic medical illness, alcohol abuse, anabolic steroid use, or reproductive dysfunction.

After screening 42 men, 18 were excluded from completion of the treatment phase of the study. Four men were excluded because of baseline low sperm concentration, two men because of prescription psychotropic medication use, one man for dyslipidemia, one man for significant hypertension and hematuria, one man for alcohol abuse, and one man did not want weekly im shots. Seven men discontinued the study because they were unable to attend clinic regularly, and one man was discontinued because he failed to comply with the protocol.

\section{Experimental Design}

After meeting the screening criteria, 24 subjects were entered into a 3-month control period during which monthly baseline serum hormone levels and biweekly seminal fluid analyses were performed while no hormones were administered. At the end of the control period, each subject entered a 6-month administration period and was randomized in a single-blind, balanced design to one of three groups: 6 months of $150 \mu \mathrm{g}$ of oral DSG daily plus $50 \mathrm{mg}$ of im testosterone enanthate weekly (DSG 150-T 50 group; $\mathrm{n}=9$ ), or 6 months of $150 \mu \mathrm{g}$ or $300 \mu \mathrm{g}$ of oral DSG daily plus 100 $\mathrm{mg}$ of im testosterone enanthate weekly (DSG 150-T 100 and DSG 300-T 100 groups; $\mathrm{n}=7$ and 8, respectively). Each subject received either one active tablet of $150 \mu \mathrm{g}$ and one placebo DSG or two active tablets depending on his group assignment. Each subject kept a medication log book that was reviewed monthly.

For purposes of comparing efficacy and side effects of DSG plus testosterone with a progestin-androgen combination regimen that was previously shown to be a promising male contraceptive regimen, we included data from groups of normal, healthy young men (T-alone group and LNG 125-T 100 group; $\mathrm{n}=18$ for each group) who were assessed in identical studies $(7,8)$ to the one reported here except that they received testosterone enanthate $(100 \mathrm{mg} / \mathrm{wk} \mathrm{im})$ alone or in combination with low-dosage LNG (125 $\mu \mathrm{g} / \mathrm{d}$ po). After the treatment period, all subjects entered a recovery period that extended until two consecutive sperm concentrations were within the individual's control range.

All subjects gave informed consent, and the studies were approved by the University of Washington Human Subjects Review Committee and the Veterans Affairs Puget Sound Health Care System Research and Development Committee.

\section{Measurements}

Monthly interviews and physical examinations were performed by physicians throughout the study. Seminal fluid analysis was performed every 2 weeks on samples obtained by masturbation after 48 hours of abstinence. Monthly blood samples were obtained for measurement of serum testosterone, FSH, and LH levels. During treatment, blood samples were drawn immediately before the administration of testosterone. Monthly nadir and peak serum DSG levels were measured from samples taken immediately before and 1 hour after DSG ingestion. Monthly urinalysis and measurement of blood counts and serum electrolytes, creatinine, and hepatic function tests were performed. A lipid panel (total cholesterol, HDL, apoprotein A-I [apoA-I] levels, low-density lipoprotein [LDL], and triglycerides levels) was performed on serum obtained after a 12-hour fast once during the control period, once after the 3rd and 6th months of the treatment period, and once during the recovery period. 


\section{Hormone and Lipid Assays}

Serum testosterone levels were measured by radioimmunoassay with use of reagents from the WHO-matched reagent program by methods previously described (10). The assay sensitivity was $0.017 \mathrm{nmol} / \mathrm{L}$; the intraassay and interassay variabilities were $5.1 \%$ and $9.8 \%$, respectively. Serum FSH and LH levels were measured in the Delfia immunofluorometric assay (Wallac Oy, Turku, Finland). The sensitivities of the Delfia assays were $<0.016 \mathrm{IU} / \mathrm{L}$ and $<0.018$ IU/L for FSH and LH, respectively. Intraassay coefficients of variation were $2.3 \%$ for FSH and $2.8 \%$ for $\mathrm{LH}$. Interassay coefficients of variation were $4.0 \%$ for FSH and $5.0 \%$ for LH. DSG levels were assayed by radioimmunoassay at NV Organon. All samples from each individual were measured in the same hormone assays to avoid interassay variability. Hormone sensitivities were determined by the first point discernible from zero on standard curves.

Lipid analyses were performed on freshly prepared (unfrozen) plasma samples that were centrifuged within hours of collection. Total plasma cholesterol and triglycerides were measured enzymatically at the Northwest Lipid Research Clinic (Seattle, WA) on the Abbott Spectrum multichromatic instrument (Abbott Laboratories, North Chicago, IL); cholesterol was measured by a Trinder-type method and triglycerides by a ultraviolet light method (11). High-density lipoprotein cholesterol was separated from plasma by precipitation with dextran sulfate-magnesium (12). Low-density lipoprotein was calculated indirectly by the Friedewald equation (13).

\section{Sperm Counts}

Azoospermia was defined as two or more consecutive sperm counts of zero, and severe oligoazoospermia was defined as two or more consecutive counts of $<1 \times 10^{6}$ spermatozoa/mL. Sperm count recovery was defined as the first of three normal sperm counts $\left(>20 \times 10^{6} / \mathrm{mL}\right)$ with at least one value equal to the subject's mean baseline count. Sperm counts were determined by a Coulter counter (Coulter Electronics, Inc., Hialeah, FL), and concentrations of $<15 \times$ $10^{6} / \mathrm{mL}$ were confirmed by direct determination with use of a hemocytometer $(14,15)$. Sperm motility assessment was performed according to the WHO laboratory manual for the examination of human semen and sperm-cervical mucus interaction (16).

\section{Statistical Analysis}

The groups were compared by ANOVA with a Duncan's post hoc test. When baseline differences were found between the four groups, the data were also analyzed by analysis of covariance (ANCOVA), but the results yielded from the ANCOVA did not differ from the ANOVA. Comparisons that included the historical controls (testosterone alone and LNG 125-T 100) and the other four groups were performed with use of unpaired $t$ tests. The analyses for severe oligoazoospermia and azoospermia were performed with use of a Kaplan-Meier curve with a log rank analysis and $\chi^{2}$ analysis.

\section{RESULTS}

\section{Baseline Characteristics}

The three groups were similar in all of their baseline clinical and biochemical characteristics except that the DSG 150-T 100 group had significantly higher serum total cholesterol, triglyceride, and hemoglobin levels than the other two DSG-T groups (Table 1).

All but 2 of the 24 men completed the full 6 months of treatment. For reasons unrelated to the study, two of the subjects in the DSG 150-T 50 group discontinued after 18 and 22 weeks of treatment, respectively. The first DSG 150-T 50 subject was azoospermic on his penultimate seminal fluid sample, but his final sample obtained during treatment was $8.7 \times 10^{6} / \mathrm{mL}$. He was included in the analysis for severe oligoazoospermia and was considered a nonresponder because he did not meet strict criteria of two consecutive sperm counts of $<1 \times 10^{6} / \mathrm{mL}$. The other DSG 150-T 50 subject had two consecutive samples of $0.1 \times 10^{6} \mathrm{sperm} / \mathrm{mL}$ before ceasing to submit seminal fluid specimens. He was included in the analysis for severe oligoazoospermia as a responder. Neither subject was included in the analysis for severe azoospermia.

\section{Response to Treatment}

\section{Sperm Counts}

The DSG groups and two historical controls (LNG 125-T 100 and testosterone alone) were analyzed according to whether they achieved severe oligoazoospermia $\left(<1 \times 10^{6}\right.$ sperm $/ \mathrm{mL}$ ) or azoospermia. Severe oligoazoospermia and azoospermia were achieved more uniformly in the groups who received progestin plus testosterone 100 (DSG 150-T 100, DSG 300-T 100, and LNG 125-T 100) than the DSG 150-T 50 or T-alone groups (Table 2 and Figs. 1 and 2). All the men in the DSG 150-T 100 and seven of eight men in the DSG 300-T 100 groups became azoospermic. The only man in the DSG 300-T 100 group who did not become azoospermic suppressed to $3 \times 10^{6} / \mathrm{mL}$ and $0.2 \times 10^{6} / \mathrm{mL}$ spermatazoa on his last two samples during treatment; he was classified as a nonresponder. The groups who received progestin plus testosterone 100 (DSG 150-T 100, DSG 300-T 100, and LNG 125-T 100) all suppressed to severe oligoazoospermia more rapidly than the DSG 150 -T 50 or testosterone-alone groups (median time to severe oligoazoospermia: 17, 11, 7, 10 , and 10 weeks in the testosterone-alone, DSG 150-T 50, DSG 150-T 100, DSG 300-T 100, and LNG 125-T 100 groups, respectively; Figs. 1 and 2). Sperm counts returned to baseline levels in the subjects who completed the recovery phase of the trial. 
Baseline clinical and biochemical parameters for 24 healthy young men randomized to three different desogestrel plus testosterone regimens.

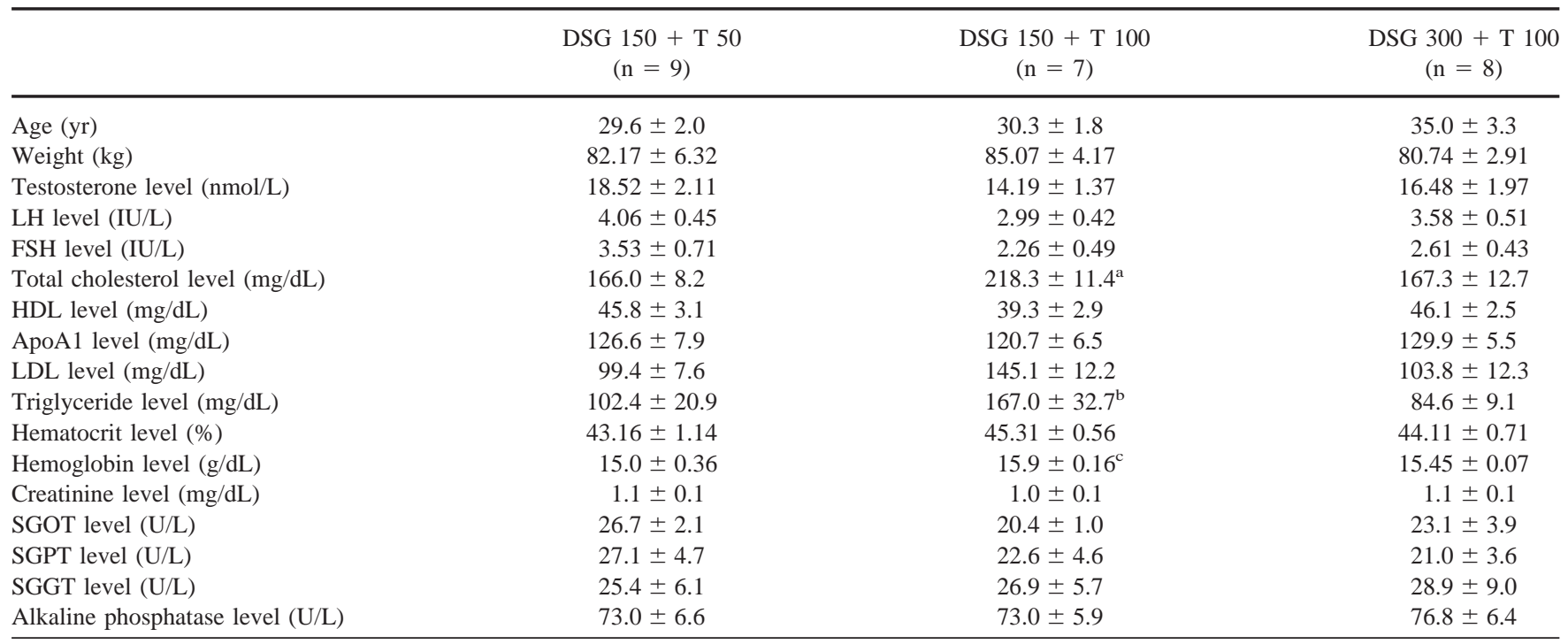

Note: Values are means \pm SEM. DSG $=$ desogestrel; $\mathrm{T}=$ testosterone.

${ }^{\text {a }} P<.05$ compared with DSG150-T50 and DSG300-T100.

${ }^{\mathrm{b}} P<.05$ compared with DSG300-T100.

${ }^{\mathrm{c}} P<.05$ compared with DSG150-T50.

Anawalt. Desogestrel plus testosterone suppresses spermatogenesis. Fertil Steril 2000.

\section{Hormones}

Testosterone levels increased during treatment in all DSG groups except the DSG 150-T 50 group whose levels decreased during treatment (percentage increase in DSG 150-T 100 and DSG 300-T 100: $47 \% \pm 26 \%, 17 \% \pm 20 \%$ increase, but there was a $20 \% \pm 10 \%$ decrease from baseline testosterone levels in the DSG 150-T 50 group; $P=\mathrm{NS}$ ). All groups had significant suppression of FSH and LH levels during the treatment period compared to baseline $(P<.05)$, but both FSH and LH levels were significantly more suppressed during treatment in all three groups of men who received T-100 plus a progestin compared to the DSG 150-T 50 group or the testosterone alone group $(P<.05$; Fig. 3$)$. Serum levels of testosterone, FSH, and $\mathrm{LH}$ all recovered to baseline levels during the recovery period ( $P=\mathrm{NS}$ compared to baseline for all groups). Peak serum DSG levels measured 1 hour after ingestion of the DSG were highest in the DSG 300-T 100 during the treatment period (peak DSG levels in treatment month 6: $801 \pm 79,652 \pm 71,1,099 \pm$ $144 \mu \mathrm{g} / \mathrm{L}$ in the DSG 150-T 50, DSG 150-T 100, and DSG 300-T 100 groups, respectively). Trough serum DSG levels drawn immediately before DSG ingestion also were highest in the DSG 300-T 100 group (trough DSG levels in treatment month 6: $217 \pm 73,132 \pm 29,323 \pm 81 \mu \mathrm{g} / \mathrm{L}$ in the DSG 150-T 50, DSG 150-T 100, and DSG 300-T 100 groups, respectively).

\section{Lipids}

Total cholesterol levels decreased slightly during treatment in the three DSG groups (percent decrease from baseline: $2.5 \% \pm 4.0 \%, 10.6 \% \pm 5.0 \%, 1.5 \% \pm 5.25 \%$ in the DSG 150-T 50, DSG 150-T 100, and DSG 300-T 100

\section{TABLE 2}

Effectiveness of suppressing sperm counts to levels associated with effective male contraception.

\begin{tabular}{|c|c|c|c|c|c|}
\hline Sperm count & T 100 alone & DSG $150+$ T 50 & DSG $150+$ T 100 & DSG $300+$ T 100 & LNG $125+$ T 100 \\
\hline Azoospermia & $33 \%(6 / 18)^{\mathrm{a}}$ & $57 \%(4 / 7)$ & $100 \%(7 / 7)$ & $88 \%(7 / 8)$ & $61 \%(11 / 18)$ \\
\hline
\end{tabular}

${ }^{a} P<.05$ compared with DSG 150-T 100, DSG 300-T 100, and LNG 125-T 100 groups.

Anawalt. Desogestrel plus testosterone suppresses spermatogenesis. Fertil Steril 2000. 


\section{FIGURE 1}

Rapidity of suppression to severe oligoazoospermia $\left(<1 \times 10^{6}\right.$ spermatozoa/mL) in normal men who received 6 months of testosterone alone or testosterone plus various progestins. The groups receiving testosterone $100 \mathrm{mg} / \mathrm{wk}$ plus DSG or LNG suppressed faster than the groups receiving testosterone alone or testosterone $50 \mathrm{mg} / \mathrm{wk}$ plus low-dosage DSG (150 $\mu \mathrm{g} / \mathrm{d}$ ). ${ }^{*} P<.05$ for DSG 150-T 100 and DSG 300-T 100 compared to testosterone alone and testosterone 50- DSG 150 by week 10.

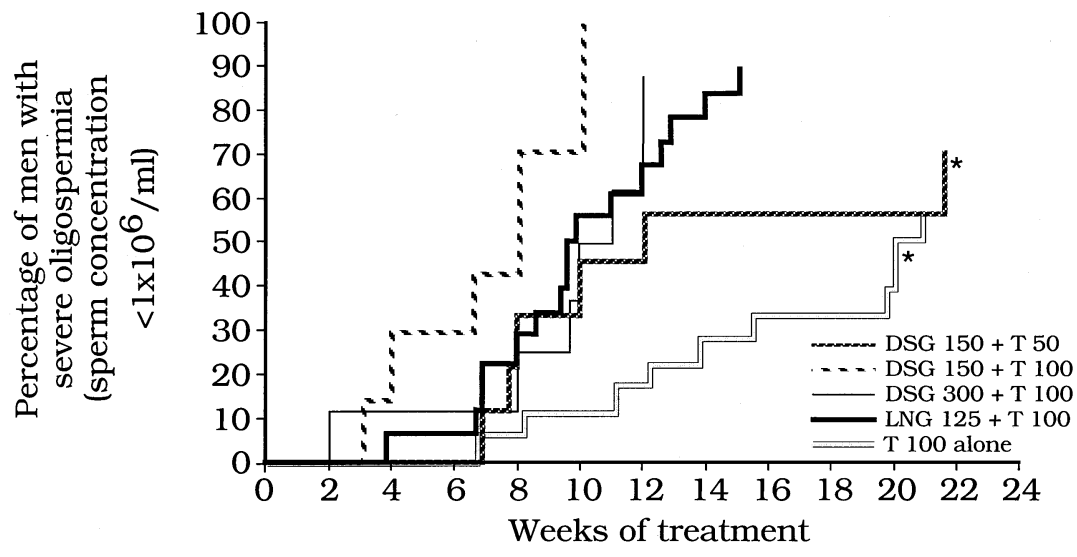

Anawalt. Desogestrel plus testosterone suppresses spermatogenesis. Fertil Steril 2000.

groups, respectively; $P=$ NS from baseline and between groups). High-density lipoprotein cholesterol levels decreased in all five groups studied, but the decrease in HDL was greatest in the high-dosage DSG group (percent de- crease from baseline: $4.0 \% \pm 4 \%, 4.8 \% \pm 11.4 \%, 10.0 \% \pm$ $4.7 \%, 20.3 \% \pm 3.7 \%$, and $13.4 \% \pm 3.7 \%$ in the testosterone-alone, DSG 150-T 50, DSG 150-T 100, DSG 300-T 100, and LNG 125-T 100 groups, respectively; Fig. 4). Only the

\section{FIGURE 2}

Rapidity of suppression of sperm concentrations from baseline in normal men who received 6 months of testosterone alone or testosterone plus various progestins. The group receiving testosterone $100 \mathrm{mg} / \mathrm{wk}$ plus DSG or LNG suppressed faster than the testosterone alone or DSG $150-\mathrm{T} 50$ groups $(P<.05$ by week 10$)$.

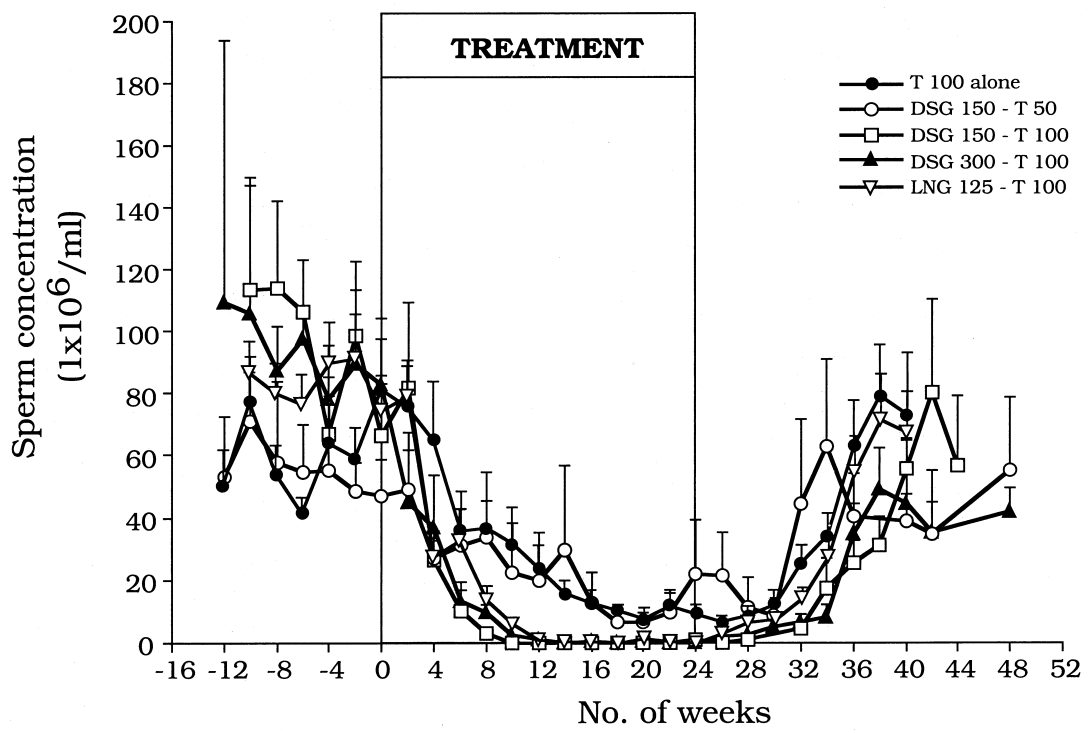

Anawalt. Desogestrel plus testosterone suppresses spermatogenesis. Fertil Steril 2000. 


\section{FIGURE 3}

Levels of serum gonadotropins (mean \pm SEM) measured at month 5 or 6 in normal men administered 6 months of testosterone alone or testosterone plus various progestins. The combination of testosterone $100 \mathrm{mg} / \mathrm{wk}$ plus DSG or LNG suppressed circulating gonadotropin levels significantly more than testosterone alone or DSG 150-T 50 groups. Values are means \pm SEM. ${ }^{*} P<.05$ compared to DSG 150-T 100, DSG 300-T 100, and LNG 125-T 100.
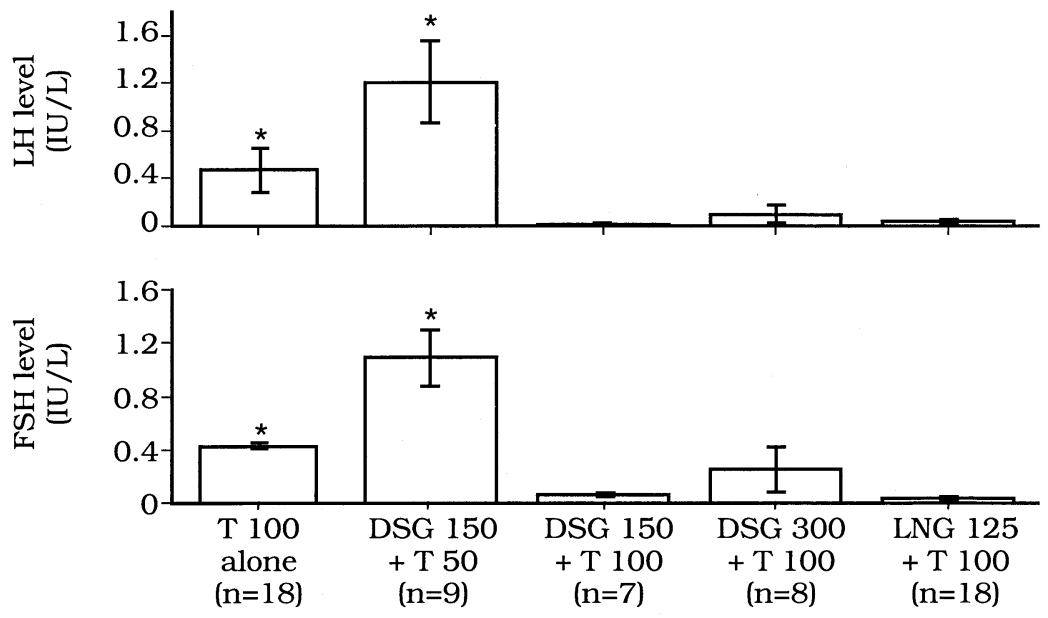

Anawalt. Desogestrel plus testosterone suppresses spermatogenesis. Fertil Steril 2000.

changes in the last two groups (DSG 300-T 100 and LNG 125-T 100) were statistically significantly decreased from baseline $(P<.05)$. The apoA-I levels tended to decrease in a pattern similar to HDL cholesterol (percent decrease from baseline: $2.6 \% \pm 11.7 \%, 12.2 \% \pm 4.0 \%, 15.1 \% \pm 4.7 \%$, and $10.4 \% \pm 3.4 \%$ in the DSG $150-\mathrm{T} 50$, DSG 150-T 100, DSG 300-T 100, and LNG 125-T 100 groups, respectively; $P=$ NS). Low-density cholesterol and fasting triglyceride levels did not change significantly throughout the study. All lipoprotein profiles returned to baseline during the recovery period.

\section{Hematologic Profiles, Blood Chemistries, and Urinalyses}

Serum hemoglobin levels did not change significantly from baseline during the treatment and recovery periods, although in one subject in the DSG 300-T 100 group whose

\section{FIGURE 4}

Suppression of HDL levels (percentage change from baseline at month 5 or 6 ) in normal men administered 6 months of testosterone alone or testosterone plus various progestins. High-density lipoprotein cholesterol levels were significantly suppressed compared to baseline in the DSG 300-T 100 and LNG 125-T 100 groups. Values are means \pm SEM. ${ }^{\star} P<.05$ compared to testosterone alone.

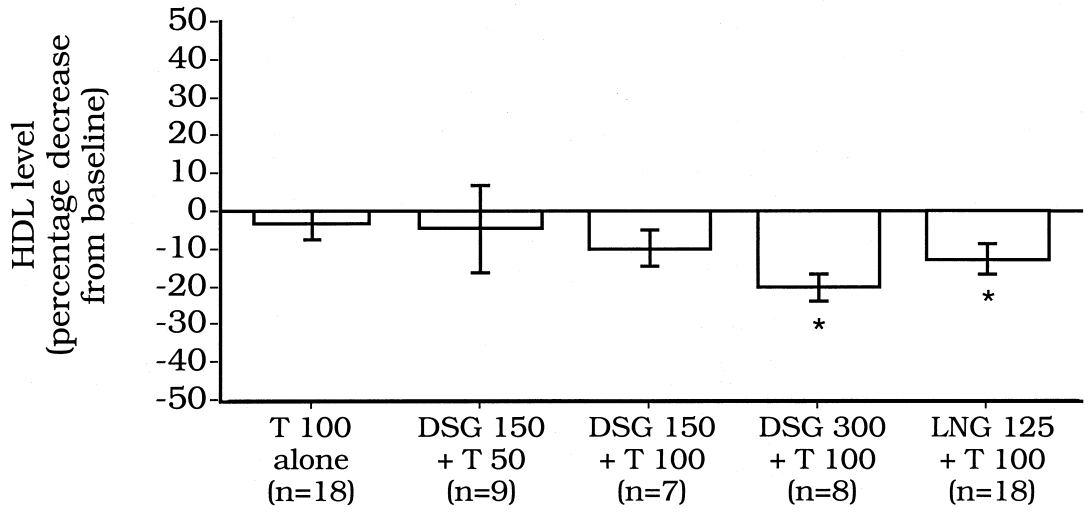

Anawalt. Desogestrel plus testosterone suppresses spermatogenesis. Fertil Steril 2000. 
Weight gain (change in kilograms from baseline at month 5 or 6 ) in normal men administered 6 months of testosterone alone or testosterone plus various progestins. Weight gain tended to increase compared to baseline in all groups and showed a statistically significant change in the DSG 150-T 100, DSG 300-T 100, and LNG 125-T 100 groups. Values are means \pm SEM. ${ }^{\star} P<.05$ compared to baseline.

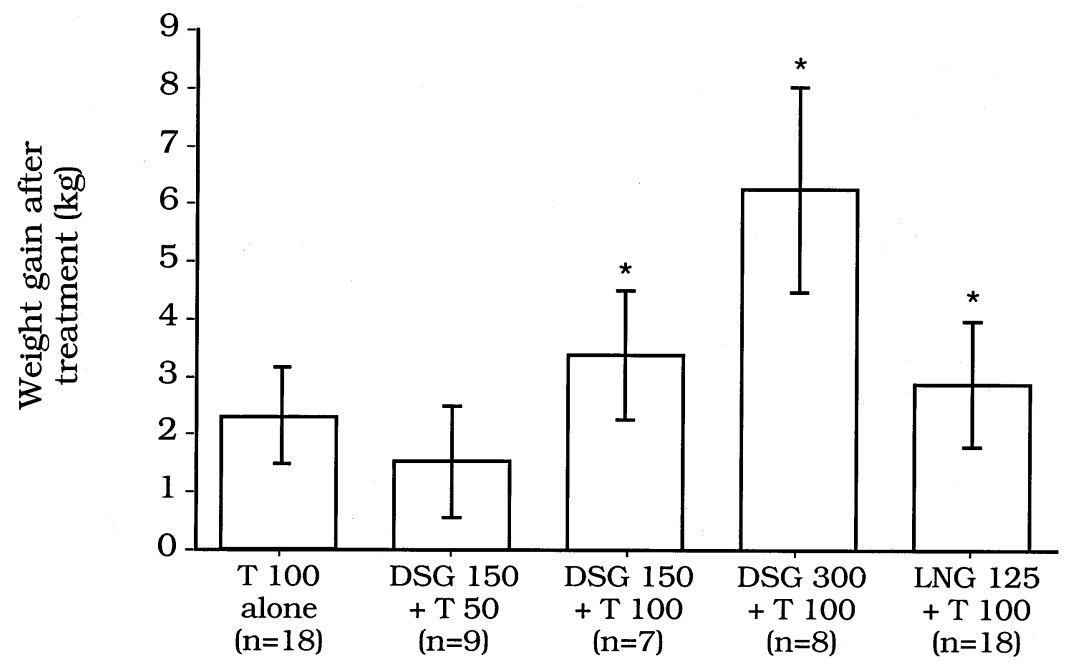

Anawalt. Desogestrel plus testosterone suppresses spermatogenesis. Fertil Steril 2000.

baseline serum hemoglobin level was $16.4 \mathrm{~g} / \mathrm{dL}$, the level rose to $18 \mathrm{~g} / \mathrm{dL}$ at the end of the treatment period. Blood chemistries including bilirubin, liver transaminases, and alkaline phosphatase remained normal and without significant change from baseline during the treatment and recovery periods. No subject developed significant changes in urinalysis during the treatment period.

\section{Weight Gain, Gynecomastia, and Acne}

Body weight tended to increase during hormone administration in all five groups (Fig. 5). The weight increase in the three progestin plus testosterone 100 groups was statistically significant compared to baseline values $(P<.05)$. Body weight decreased to baseline during the recovery period. Three men ( 1 in each DSG group) developed acne during the treatment phase of the study and were treated with topical creams or systemic antibiotics. No man developed significant gynecomastia.

\section{DISCUSSION}

This study shows that the combination of DSG plus low-dosage testosterone (100 mg/wk) uniformly suppresses spermatogenesis to levels associated with effective contraception $(3,4)$. Although the numbers of men represented in this study are small, virtually all men (94\%) who were given either 150 or $300 \mu \mathrm{g}$ of DSG daily plus $100 \mathrm{mg}$ of testosterone enanthate weekly became azoospermic, and the one subject who did not become azoospermic suppressed to
$0.2 \times 10^{6} / \mathrm{mL}$ by the end of treatment. Based on data from the WHO study, these results suggest that the combination of DSG plus low-dosage testosterone would confer contraceptive efficacy similar to reversible female methods such as injectable or oral hormonal contraceptives (2-4).

We compared the combination of DSG plus testosterone to the combination of LNG plus testosterone because the latter has been shown to induce a substantial suppression of spermatogenesis and might be a potential male hormonal contraceptive regimen $(7,8)$. In our present study, the regimens that contained either 150 or $300 \mu \mathrm{g}$ of DSG daily plus $100 \mathrm{mg}$ of testosterone weekly were at least as effective in uniformly suppressing circulating gonadotropin levels and inducing severe oligoazoospermia and seemed to more effectively induce azoospermia than the same dosage of 125 $\mu \mathrm{g}$ of LNG daily plus $100 \mathrm{mg}$ of testosterone weekly. Although the numbers are small in our study, DSG 150-T 100 and DSG 300-T 100 were also significantly more effective in suppressing circulating gonadotropins and, therefore, in suppressing spermatogenesis than the lower-dosage regimen of DSG 150-T 50. Finally, there seemed to be a dosedependent effect on HDL cholesterol and weight by DSG with the higher dosage of DSG, causing twice as much HDL cholesterol suppression and weight gain as the lower dosage of DSG or the LNG groups.

The DSG plus testosterone regimens were safe and well tolerated. One subject in the high-dosage DSG group developed a mild erythrocytosis at the end of treatment, but his 
hematologic profile normalized during recovery. Otherwise, the major side effect was mild acne. No subject dropped out of the study during the treatment phase.

A complementary study (Wu et al.) (17), which was done as a part of a two-center dose-finding study with our study, showed similar efficacy. Wu's study, which differed slightly in design from our study, included three groups of men who were administered $300 \mu \mathrm{g}$ of oral DSG plus 50 or $100 \mathrm{mg}$ of IM testosterone enanthate weekly or $150 \mu \mathrm{g}$ of oral DSG daily plus $100 \mathrm{mg}$ of im testosterone enanthate weekly for 6 months. In Wu's study, 92\% (22 of 24) of all of the subjects receiving DSG plus testosterone became severely oligoazoospermic and $79 \%$ became azoospermic.

Comparison with our study is inexact because their study included a 3-week period of DSG alone at the beginning of the treatment period, and their study used some different dosages of DSG and testosterone. However, the DSG 150-T 100 group was common to both studies. On the basis of the combined data from the two studies, $80 \%$ (12 of 15) of the DSG 150-T 100 became azoospermic, and 100\% (15 of 15) suppressed to sperm counts of $<1 \times 10^{6} / \mathrm{mL}$. In the two studies, all combinations of the DSG plus testosterone regimens studied except the combination of DSG 150-T 50 seemed to be significantly better than a testosterone-alone regimen for rapidly and uniformly achieving severe oligoazoospermia or azoospermia.

Combinations of progestins and androgens seem to be promising candidates for successful male contraceptive regimens (6). A successful progestin-androgen combination must uniformly suppress circulating gonadotropin levels and spermatogenesis, provide adequate androgen effect to maintain androgen-dependent functions, but minimize adverse effects of androgen excess such as HDL suppression that might be associated with increased risk of cardiovascular disease (18). The use of a progestin with antiandrogenic properties, such as cyproterone acetate, in combination with low-dosage testosterone has been shown in a small study to provide these characteristics (19). Desogestrel is an interesting new possibility to add to progestins such as LNG and cyproterone acetate for developing a range of effective, safe, and reversible male hormonal contraceptives. Further studies could be performed with lower dosages of DSG in combination with low-dosage testosterone dosages to establish the best regimen that uniformly suppresses circulating gonadotropins and spermatogenesis while causing minimal or no effects of androgenic excess such as HDL cholesterol suppression, weight gain, or acne.

Acknowledgments: The authors thank the following individuals for their assistance in completing this study: Elaine Rost and Jennifer Bullock for coordinating the project, Consuelo Pete, Dorothy McGuinness, Elizabeth Van Gaver, and Arlen Sarkissian for their technical assistance; Liza Noonan for biostatistical analysis; Drs. Carrie Bagatell, David Gruenewald, and Alvin Paulsen for their help in examining subjects; Dr. Santica Marcovina for measurement of apoprotein levels and NV Organon, OSS, Netherlands, for providing desogestrel and performing the desogestrel assays.

\section{References}

1. World Health Organization Task Force on Methods for the Regulation of Male Fertility. Contraceptive efficacy of testosterone-induced azoospermia in normal men. Lancet 1990;336:955-9.

2. World Health Organization Task Force on Methods for the Regulation of Male Fertility. Contraceptive efficacy of testosterone-induced azoospermia and oligo-azoospermia in normal men. Fertil Steril 1996; 65:821-9.

3. Amory JK, Bremner WJ. The use of testosterone as a male contraceptive. In: S Bhasin, editor. The therapeutic role of androgens. Baillieres Clinics in Endocrinology 1998;7(12):1977-85.

4. Wu FCW. Male contraception. Baillieres Clin Obstet Gynaecol 1996; 10:1-23.

5. Bagatell CJ, Herman JR, Matsumoto AM, Rivier JE, Bremner WJ. Metabolic and behavioral effects of high-dose, exogenous testosterone in healthy men. J Clin Endocrinol Metab 1994;79:561-7.

6. Meriggiola MC, Bremner WJ. Progestogen-androgen combination regimens for male contraception. J Androl 1997;18:240-3.

7. Bebb RA, Anawalt BD, Christensen RB, Paulsen CA, Bremner WJ, Matsumoto AM. Combined administration of levonorgestrel and testosterone induces more rapid and effective suppression of spermatogenesis than testosterone alone: a promising male contraceptive approach. J Clin Endocrinol Metab 1996;81:757-62.

8. Anawalt BD, Bebb RA, Bremner WJ, Matsumoto AM. A lower-dosage levonorgestrel and testosterone combination effectively suppresses spermatogenesis and circulating gonadotropin levels with fewer metabolic effects than higher-dosage combinations. J Androl 1999;20:407-14.

9. Darney PD. The androgenicity of progestins. Am J Med 1995;98: 104-10.

10. Matsumoto AM, Paulsen CA, Hopper BR, Rebar RW, Bremner WJ. Human chorionic gonadotropin and testicular function: stimulation of testosterone, testosterone products, and sperm production despite high estradiol levels. J Clin Endocrinol Metab 1983;56:720-8.

11. Warnick GR, Benderson J, Albers JJ. Dextran sulfate $\mathrm{Mg}^{2+}$ precipitation procedure for quantitation of high-density-lipoprotein cholesterol. Clin Chem 1982;28:1379-88.

12. Warnick GR. Enzymatic methods for the quantification of lipoprotein lipids. In: Albers JJ, Segrest JP, editors. Methods in enzymology. Orlando: Academic Press, 1982;129:101-23.

13. Friedewald WT, Levy RI, Fredrickson DS. Estimation of the concentration of low-density lipoprotein cholesterol in plasma without the use of the preparative centrifuge. Clin Chem 1972;18:499-501.

14. Bremner WJ, Matsumoto AM, Sussman AM, Paulsen CA. Folliclestimulating hormone and human spermatogenesis. J Clin Invest 1981; 68:1044-52.

15. Gordon DL, Moore DL, Thorslund T, Paulsen CA. The determination of size and concentration of human sperm with an electronic particle counter. J Lab Clin Med 1965;65:506-12.

16. World Health Organization. Laboratory manual for the examination of human semen and sperm-cervical mucus interaction. 3rd ed. Cambridge: Cambridge University Press, 1993;7-8.

17. Wu FCW, Balasubramanian R, Mulders TMT, Coelingh-Bennink HJT. Oral progestin combined with testosterone as a potential male contraceptive: additive effects between desogestrel and testosterone in suppression of spermatogenesis, the pituitary-testicular axis, and lipid metabolism. J Clin Endocrinol Metab 1999;84:112-22.

18. National Institutes of Health Consensus Development Panel. Triglyceride, high-density lipoprotein, and coronary heart disease. J Am Med Assoc 1993;269:505-11.

19. Meriggiola MC, Bremner WJ, Paulsen CA, Valdiserri A, Incorvaia L, Motra R, et al. A combined regimen of cyproterone acetate and testosterone enanthate as a potentially highly effective male contraceptive. J Clin Endocrinol Metab 1996;81:3018-23. 